\title{
MINIMAL THREEFOLDS OF SMALL SLOPE AND THE NOETHER INEQUALITY FOR CANONICALLY POLARIZED THREEFOLDS
}

\author{
Meng Chen
}

\begin{abstract}
Assume that $X$ is a smooth projective 3 -fold with ample $K_{X}$. We study a problem of Miles Reid to prove the inequality

$$
K_{X}^{3} \geq \frac{2}{3}\left(2 p_{g}(X)-5\right),
$$

where $p_{g}(X)$ is the geometric genus. This inequality is sharp according to known examples of M. Kobayashi. We also birationally classify arbitrary minimal 3-folds of general type with small slope.
\end{abstract}

\section{Introduction}

We work over an algebraically closed field $k$ of characteristic 0 .

On an irreducible complete curve $C$ (may be singular), one has $\operatorname{deg}\left(K_{C}\right) \geq$ $2 g(C)-2$ where $g(C)$ is the geometric genus of $C$.

This inequality has a 2-dimensional analogue which is the famous "Noether's inequality" (see [16]). Explicitly, on a minimal surface $S$ (with RDP singularities) of general type, one has

$$
K_{S}^{2} \geq 2 p_{g}(S)-4
$$

where $p_{g}(S):=h^{0}\left(S, K_{S}\right)$ is the geometric genus of $S$. Together with the Bogomolov-Miyaoka-Yau inequality (cf. [15], [25]): $K_{S}^{2} \leq 9 \chi\left(\mathcal{O}_{S}\right)$, they have ever played very important roles in surface theory (for instance the surface geography: see [2], [6], [7], [18] and [23] etc.).

The importance of the Noether inequality in mind, Miles Reid first asked the question seeking for a 3-dimensional analogue in early 1980's. Since then, there have been many papers which give effective Noether type of inequalities either in the form $K^{3} \geq a \chi+b$ (see [17] and [1] etc.) or for restricted objects (see [13] and [3] etc.). We mention here that an effective linear inequality in terms of $\chi$ seems to be impossible because $\chi$ could be both positive and negative for a general 3-fold of general type. It is Kobayashi's interesting examples ([13]) that shows the naive inequality $K^{3} \geq 2 p_{g}-6$ (in 3 -dimensional case) is not correct in general. Thus it becomes more interesting what the 3-dimensional Noether

Received July 14, 2004.

This paper is supported by The Institute of Mathematical Sciences, The Chinese University of Hong Kong. The project is also supported by the National Natural Science Foundation of China (No.10131010), Shanghai Scientific \& Technical Commission (Grant 01QA14042). 
inequality is even under very restricted conditions. Such a "Noether inequality" is by all means important to the 3 -fold geography (see [10]).

The aim of this paper is to present a Noether inequality for canonically polarized threefolds and to give a classification to arbitrary minimal 3-folds of general type with small slope. Our main results are as follows.

Theorem 1.1. Let $X$ be a smooth projective threefold with ample canonical divisor $K_{X}$. Then

$$
K_{X}^{3} \geq \frac{2}{3}\left(2 p_{g}(X)-5\right) .
$$

Theorem 1.2. Let $X$ be a minimal projective Gorenstein 3-fold of general type with canonical singularities. Assume

$$
K_{X}^{3}<2 p_{g}(X)-6
$$

Then $X$ is fibred by curves of genus 2 .

Theorem 1.3. Let $X$ be a projective minimal 3-fold with only canonical singularities. Assume

$$
K_{X}^{3}<\frac{1}{2}\left(3 p_{g}(X)-5\right)
$$

(and $p_{g}(X) \notin[2,11]$ ). Then $X$ must be fibred by curves of genus 2 .

Further classifications to restricted minimal 3-folds are presented as an interesting application.

Corollary 1.4. Let $X$ be a smooth projective 3-fold with ample $K_{X}$. Assume

$$
K_{X}^{3}<\frac{3}{2} p_{g}(X)-\frac{9}{2}
$$

Then $X$ must be canonically fibred by curves of genus 2.

Theorem 1.1 is sharp according to M. Kobayashi's interesting examples ([13]) which say that there are canonically polarized 3 -folds with infinite number of configurations of invariants $\left(K^{3}, p_{g}\right)$ satisfying the equality: $K^{3}=\frac{2}{3}\left(2 p_{g}-5\right)$. Again due to Kobayashi's examples, Theorem 1.2 and Theorem 1.3 are not empty and they are parallel to surface case (see [21] and [24]). We do not know, however, whether both are optimal.

Based on our previous paper ([3]), and in order to prove Theorem 1.1, we need to treat the most difficult case, i.e. when $X$ is canonically fibred by surfaces of general type with $\left(c_{1}^{2}, p_{g}\right)=(1,2)$, through new methods. The new observation of this paper is that we may choose a special embedded resolution to the given polarized 3-fold and then successfully apply the Kawamata-Viehweg vanishing theorem to $\mathbb{Q}$-divisors on both 3 -folds and surfaces to estimate the dimension of those cohomological groups in question. We bound the $K_{X}^{3}$ from below by studying the bicanonical system rather than in the traditional way.

This note was written while I was visiting the Institute of Mathematical Sciences, the Chinese University of Hong Kong. I would like to thank Eckart 
Viehweg for his patient explaining my frequent email queries. Thanks are also due to Kang Zuo for effective discussions and for his hospitality. Finally I appreciate many helps from both Keiji Oguiso and Seunghun Lee.

\section{Proof of Theorem 1.1 and Theorem 1.2}

In this section, We work on minimal 3-folds of canonical index 1. According to the Mori minimal model theory ([12], [14] and [19] etc.), one may take $X$ to be a minimal projective threefold with invertible canonical sheaf $\omega_{X}=\mathcal{O}_{X}\left(K_{X}\right)$ and with locally factorial terminal singularities. From the expression of the inequality in Theorem 1.1. One may assume $p_{g}(X) \geq 3$.

2.1. Notations. We study the canonical map $\varphi_{1}$ which is usually a rational map. Take the birational modification $\pi: X^{\prime} \rightarrow X$, according to Hironaka, such that

(i) $X^{\prime}$ is smooth;

(ii) the movable part of $\left|K_{X^{\prime}}\right|$ is basepoint free.

(iii) $\pi^{*}\left(K_{X}\right)$ is linearly equivalent to a divisor supported by a divisor of normal crossings.

Denote by $g$ the composition $\varphi_{1} \circ \pi$. So $g: X^{\prime} \longrightarrow W^{\prime} \subseteq \mathbb{P}^{p_{g}(X)-1}$ is a

morphism. Let $g: X^{\prime} \stackrel{f}{\longrightarrow} B \stackrel{s}{\longrightarrow} W^{\prime}$ be the Stein factorization of $g$. We have the following commutative diagram:

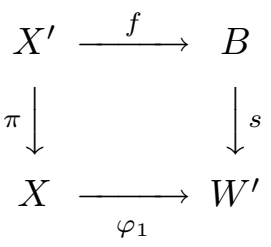

We may write

$$
K_{X^{\prime}}=\pi^{*}\left(K_{X}\right)+\bar{E}=M+\bar{Z}
$$

where $M$ is the movable part of $\left|K_{X^{\prime}}\right|, \bar{Z}$ the fixed part and $\bar{E}$ an effective divisor which is a sum of distinct exceptional divisors. Throughout we always mean $\pi^{*}\left(K_{X}\right)$ by $K_{X^{\prime}}-\bar{E}$. So, whenever we take the round up of $\alpha \pi^{*}\left(K_{X}\right)$, we always have $\left\ulcorner\alpha \pi^{*}\left(K_{X}\right)\right\urcorner \leq\ulcorner\alpha\urcorner K_{X^{\prime}}$ for all positive rational number $\alpha$. We may also write

$$
\pi^{*}\left(K_{X}\right)=M+E_{1}^{\prime}
$$

where $E_{1}^{\prime}=\bar{Z}-\bar{E}$ is actually an effective divisor.

If $\operatorname{dim} \varphi_{1}(X)=2$, we see that a general fiber of $f$ is a smooth projective curve of genus $g \geq 2$. We say that $X$ is canonically fibred by curves of genus $g$.

If $\operatorname{dim} \varphi_{1}(X)=1$, we see that a general fiber $S$ of $f$ is a smooth projective surface of general type. We say that $X$ is canonically fibred by surfaces with invariants $\left(c_{1}^{2}\left(S_{0}\right), p_{g}(S)\right)$, where $S_{0}$ is the minimal model of $S$. 
2.2. The case $\operatorname{dim}(B)=3$. One has already the inequality

$$
K_{X}^{3} \geq 2 p_{g}(X)-6
$$

according to Kobyashi ([13]) even for a general minimal 3-fold of general type.

For reader's convenience, we reformulate our known results in [3] in the case $\operatorname{dim}(B) \leq 2$.

Theorem 2.3. (Theorem 4.1 of [3]) Let $X$ be a minimal projective Gorenstein 3-fold of general type with only locally factorial terminal singularities. Then we have

(i) If $\operatorname{dim} \varphi_{1}(X)=2$, i.e., $X$ is canonically fibred by curves of genus $g$, then

$$
K_{X}^{3} \geq\left\ulcorner\frac{2}{3}(g-1)\right\urcorner\left(p_{g}(X)-2\right) .
$$

(ii) If $\operatorname{dim} \varphi_{1}(X)=1$, then either $K_{X}^{3} \geq 2 p_{g}(X)-4$ or $\left(K_{S_{0}}^{2}, p_{g}(S)\right)=(1,2)$.

Theorem 2.4. (Theorem 4.3 of [3]) Let $X$ be a minimal projective smooth 3fold of general type. Suppose $\operatorname{dim} \varphi_{1}(X)=2$ and $X$ is canonically fibred by curves of genus 2 . Then

$$
K_{X}^{3} \geq \frac{2}{3}\left(2 p_{g}(X)-5\right)
$$

The inequality is sharp.

We are left to study the only case when $\operatorname{dim}(B)=1$ and $X$ is canonically fibred by surfaces with $\left(c_{1}^{2}, p_{g}\right)=(1,2)$. For this purpose, we need a little bit of preparation.

2.5. Bounding $K_{X}^{3}$ from below. For the technical reason, we must assume that $K_{X}$ is ample from now on. The inequality in Theorem 1.1 is trivial for small value of $p_{g}(X)$. One may assume $p_{g}(X) \geq 3$. Furthermore, we assume $\operatorname{dim}(B)=1$ and that a general fiber of the induced fibration $f: X^{\prime} \longrightarrow B$ is a surface with $\left(c_{1}^{2}, p_{g}\right)=(1,2)$. Set $b:=g(B)$ the geometric genus of $B$.

By Lemma 4.5 of [3], we have two cases exactly:

$$
\begin{aligned}
& q(X)=b=1 \text { and } h^{2}\left(\mathcal{O}_{X}\right)=0 \\
& q(X)=b=0 \text { and } h^{2}\left(\mathcal{O}_{X}\right) \leq 1
\end{aligned}
$$

Write

$$
\left|K_{X}\right|=|N|+Z
$$

where $Z$ is the fixed part and $N$ the movable one. Then it is obvious that

$$
N=\pi_{*}(M) \text { and } Z=\pi_{*}\left(E_{1}^{\prime}\right) .
$$

Set $F:=\pi_{*}(S)$. One may write

$$
M=\sum_{i=1}^{a} S_{i}
$$


as a disjoint union of distinct fibers of $f$, where $a=p_{g}(X)-1$ whenever $b=0$, or $a=p_{g}(X)$ otherwise. Thus we have

$$
N=\sum_{i=1}^{a} F_{i}
$$

where $F_{i}=\pi_{*}\left(S_{i}\right)$.

If $|N|$ has base points, then $F^{2}>0$ as a 1-cycle. Thus $K_{X} \cdot F^{2} \geq 2$ because it is an even number. Then it is obvious that

$$
K_{X}^{3} \geq 2 p_{g}(X)-2 .
$$

Otherwise, $|N|$ is base point free. In this case, $F$ is a nonsingular projective surface with ample $K_{F}$. Also since $\left.Z\right|_{F} \sim K_{F}$ and $K_{F}^{2}=1$, we see that $\left.Z\right|_{F}$ is an irreducible curve on the surface $F$. Because $f$ obviously factors through $X$, we denote by $f_{1}$ the induced fibration $X \longrightarrow B$. Denote by $C$ the curve $Z \cap F$. Because $C \sim K_{F}, C$ must be a curve with arithmetical genus 2. Thus $C$ must be one of the following types:

a) $C$ is smooth;

b) $C$ is an elliptic curve with exactly one node;

c) $C$ is an elliptic curve with exactly one cusp of type $x^{2}=y^{3}$;

d) $C$ is a rational curve with exactly 2 nodes;

e) $C$ is a rational curve with one node and one cusp of type $x^{2}=y^{3}$;

f) $C$ is a rational curve with exactly 2 cusps of type $x^{2}=y^{3}$;

g) $C$ is a rational curve with only one cusp of type $x^{2}=y^{5}$.

We will see later that the singularities on $C$ have strong connections with the value of $K_{X}^{3}$.

We consider the linear system $\left|K_{X^{\prime}}+\pi^{*}\left(K_{X}\right)\right|$ on $X^{\prime}$. For a general fiber $S$, denote by $\sigma: S \longrightarrow F$ the natural contraction.

Now we fix some notations. Assume $m>0$ is an integer and $0 \leq n<m$. Under the premise of $a \geq m$, we may write $a:=a_{1} m+s$ where $a_{1}>0$ is an integer and $0 \leq s<m$. One may find distinct smooth fibers $\left\{S_{k}\right\}$ such that

$$
M \sim S_{0}+\sum_{j=1}^{a-a_{1} n-1} S_{j}+\sum_{i=a-a_{1} n}^{a-1} S_{i} .
$$

Suppose the following condition $\left(^{*}\right)$ is satisfied:

(*) there is a number $r \geq 3$ such that, for all $i$ with $a-a_{1} n \leq i \leq a-1$,

$$
h^{0}\left(S_{i}, K_{S_{i}}+\left\ulcorner\left.\left(\pi^{*}\left(K_{X}\right)-\frac{n a_{1}}{m a_{1}+s} \pi^{*}(Z)\right)\right|_{S_{i}}\right)\right\urcorner \geq r .
$$

Because

$$
\pi^{*}\left(K_{X}\right)-\sum_{i=a-a_{1} n}^{a-1} S_{i}-\frac{n a_{1}}{m a_{1}+s} \pi^{*}(Z) \equiv\left(1-\frac{n a_{1}}{m a_{1}+s}\right) \pi^{*}\left(K_{X}\right)
$$

is nef and big, one has, according to the Kawamata-Viehweg vanishing theorem, the exact sequence: 


$$
\begin{aligned}
0 & \longrightarrow H^{0}\left(X^{\prime}, K_{X^{\prime}}+\left\ulcorner\pi^{*}\left(K_{X}\right)-\sum_{i=a-a_{1} n}^{a-1} S_{i}-\frac{n a_{1}}{m a_{1}+s} \pi^{*}(Z)\right\urcorner\right) \\
& \longrightarrow H^{0}\left(X^{\prime}, K_{X^{\prime}}+\left\ulcorner\pi^{*}\left(K_{X}\right)-\frac{n a_{1}}{m a_{1}+s} \pi^{*}(Z)\right\urcorner\right) \\
& \longrightarrow \oplus_{i=a-a_{1} n}^{a} H^{0}\left(S_{i}, K_{S_{i}}+\left.\left\ulcorner\pi^{*}\left(K_{X}\right)-\frac{n a_{1}}{m a_{1}+s} \pi^{*}(Z)\right\urcorner\right|_{S_{i}}\right) .
\end{aligned}
$$

Similarly because

$$
\pi^{*}\left(K_{X}\right)-\sum_{j=1}^{a-a_{1} n-1} S_{j}-\sum_{i=a-a_{1} n}^{a-1} S_{i}-\frac{a-1}{a} \pi^{*}(Z) \equiv \frac{1}{a} \pi^{*}\left(K_{X}\right)
$$

is nef and big, one has again the exact sequence:

$$
\begin{aligned}
& 0 \longrightarrow H^{0}\left(X^{\prime}, K_{X^{\prime}}+\left\ulcorner\pi^{*}\left(K_{X}\right)-\sum_{j=1}^{a-a_{1} n-1} S_{j}-\sum_{i=a-a_{1} n}^{a-1} S_{i}-\frac{a-1}{a} \pi^{*}(Z)\right\urcorner\right) \\
& \longrightarrow H^{0}\left(X^{\prime}, K_{X^{\prime}}+\left\ulcorner\pi^{*}\left(K_{X}\right)-\sum_{i=a-a_{1} n}^{a-1} S_{i}-\frac{a-1}{a} \pi^{*}(Z)\right\urcorner\right) \longrightarrow \\
& \oplus_{j=1}^{a-a_{1} n-1} H^{0}\left(S_{j}, K_{S_{j}}+\left.\left\ulcorner\pi^{*}\left(K_{X}\right)-\sum_{i=a-a_{1} n}^{a-1} S_{i}-\frac{a-1}{a} \pi^{*}(Z)\right\urcorner\right|_{S_{j}}\right) .
\end{aligned}
$$

Noting that

$$
\begin{aligned}
& K_{S_{j}}+\left.\left\ulcorner\pi^{*}\left(K_{X}\right)-\sum_{i=a-a_{1} n}^{a-1} S_{i}-\frac{a-1}{a} \pi^{*}(Z)\right\urcorner\right|_{S_{j}} \\
\geq & K_{S_{j}}+\left\ulcorner\left.\left(\pi^{*}\left(K_{X}\right)-\sum_{i=a-a_{1} n}^{a-1} S_{i}-\frac{a-1}{a} \pi^{*}(Z)\right)\right|_{S_{j}}\right\urcorner \\
= & K_{S_{j}}+\left\ulcorner\left.\frac{1}{a} \pi^{*}(Z)\right|_{S_{j}}\right\urcorner \geq K_{S_{j}},
\end{aligned}
$$

we have

$$
r_{j}:=h^{0}\left(S_{j}, K_{S_{j}}+\left\ulcorner\left.\frac{1}{a} \pi^{*}(Z)\right|_{S_{j}}\right\urcorner\right) \geq p_{g}\left(S_{j}\right)=2 .
$$

On the other hand, we have

$$
\begin{aligned}
& h^{0}\left(X^{\prime}, K_{X^{\prime}}+\left\ulcorner\pi^{*}\left(K_{X}\right)-\sum_{j=1}^{a-a_{1} n-1} S_{j}-\sum_{i=a-a_{1} n}^{a-1} S_{i}-\frac{a-1}{a} \pi^{*}(Z)\right\urcorner\right) \\
& \geq h^{0}\left(X^{\prime}, K_{X^{\prime}}+S_{0}\right) .
\end{aligned}
$$

Whenever $h^{2}\left(\mathcal{O}_{X}\right)=0$, the surjective map

$$
H^{0}\left(X^{\prime}, K_{X^{\prime}}+S_{0}\right) \longrightarrow H^{0}\left(S_{0}, K_{S_{0}}\right)
$$


gives

$$
h^{0}\left(X^{\prime}, K_{X^{\prime}}+S_{0}\right)=p_{g}(X)+2 .
$$

In this situation, we set $\delta:=2$.

Whenever $b=0$ and $h^{2}\left(\mathcal{O}_{X}\right)=1$, we have

$$
h^{0}\left(X^{\prime}, K_{X^{\prime}}+S_{0}\right) \geq p_{g}(X)+1 .
$$

Whence we set $\delta:=1$.

The above two exact sequences give

$$
P_{2}(X) \geq p_{g}(X)+\delta+\sum_{j=1}^{a-a_{1} n-1} r_{j}+a_{1} n r
$$

Since

one has

$$
P_{2}(X)=\frac{1}{2} K_{X}^{3}-3\left(1-b+h^{2}\left(\mathcal{O}_{X}\right)-p_{g}(X)\right)
$$

$$
K_{X}^{3} \geq 2\left(-2 p_{g}(X)+\delta+\sum_{i=1}^{a-a_{1} n-1} r_{j}+a_{1} n r+3 h^{2}\left(\mathcal{O}_{X}\right)-3 b+3\right) .
$$

The above inequality is a key to better inequalities provided we know all the numbers $r_{j}, r$ and $n$. We study it case by case as follows. We first present the following

Lemma 2.6. On the general fiber $S$ of $f$, denote by $D:=\left(\left.\pi^{*}(Z)\right|_{S}\right)_{\text {red }}$. Then $h^{0}\left(S, K_{S}+D\right)=2$ if and only if $D$ is supported on a rational tree.

Proof. Let $N$ be a very big natural number such that $\left\ulcorner\left.\frac{1}{N} \pi^{*}(Z)\right|_{S}\right\urcorner=D$. One has

$$
\left.\left.\pi^{*}(Z)\right|_{S} \sim \pi^{*}\left(K_{X}\right)\right|_{S} \cong \sigma^{*}\left(K_{F}\right)
$$

is nef and big. Using the Riemann-Roch and the vanishing theorem, we get

$$
h^{0}\left(S, K_{S}+D\right)=\frac{1}{2} D \cdot\left(K_{S}+D\right)+\chi\left(\mathcal{O}_{S}\right) .
$$

So $h^{0}\left(S, K_{S}+D\right)=2$ if and only if

$$
\left(K_{S}+D\right) \cdot D=-2 .
$$

Because $D$ is 1-connected and reduced, it is the obvious fact that $D$ supports on a rational tree. We are done.

2.7. The case 2.5 a), b) and c). If there is a smooth fiber $F$ on $X$ such that $C=D \cap F$ is in the case $2.5 \mathrm{a}), \mathrm{b})$ and c). Taking a smooth modification to the morphism $\left.f\right|_{D}: D \mapsto B$. One may easily see that $C$ is always among these 3 types for a general fiber $F$. Thus we have $r_{j} \geq 3$ for all $j$ by Lemma 2.6. We may take $n=0$.

Now if $b=1$, then the inequality (1) gives

$$
K_{X}^{3} \geq 2\left(p_{g}(X)-1\right) .
$$


If $b=0$ and $h^{2}\left(\mathcal{O}_{X}\right)=1$, then (1) gives

$$
K_{X}^{3} \geq 2\left(p_{g}(X)+1\right) .
$$

If $b=0$ and $h^{2}\left(\mathcal{O}_{X}\right)=0$, then (1) gives

$$
K_{X}^{3} \geq 2\left(p_{g}(X)-1\right) .
$$

We are left the situation that, for a general fiber $F$ of $f_{1}, C$ falls into the cases $2.5 \mathrm{~d}$ ) through g). For these cases, our argument depend on a special modification $\pi$.

\subsection{The rest cases.}

From now on, we may suppose that $C$ is a singular rational curve for a general fiber $F$ of $f_{1}$. We proceed our proof by considering the singularities on the surface $Z$. First of all, $Z$ must be singular along a curve. Otherwise, if $Z$ has isolated singularities, $C$ would be a smooth curve of genus 2 which contradicts to our assumption.

We hope to find a special embedded resolution of the pair $(X, Z)$ to prove Theorem 1.1.

Claim 2.9. $Z$ has at most 2 horizontal (with respect to $f_{1}$ ) irreducible singular curves and the multiplicity of any such singular curve on $Z$ is 2 .

Proof. In the process of finding the embedded resolution for $(X, Z)$, we do not care those vertical modifications supported only on finite number of fibers with regard to the fibration $f_{1}: X \longrightarrow B$. This is because those vertical modifications do not affect the behavior of $\left.\pi^{*}\left(K_{X}\right)\right|_{S}$ for a general fiber $S$ of $f$. By abuse of concepts, we call this kind of vertical modifications to be negligible.

Pick up any irreducible singular curve $G$ of $Z$ such that $f_{1}(G)=B$. Because $G$ has at most finite number of singular points, we may take a negligible modification $\pi_{0}: X_{0} \longrightarrow X$ such that $G$ is smooth upstairs. Denote by $Z_{0}$ the strict transform of $Z$. We still denote by $G$ the strict transform of $G$ upstairs. On $X_{0}$, $Z_{0}$ has a singular curve along $G$ and $G$ is a smooth curve.

Let $\pi_{1}: X_{1} \longrightarrow X_{0}$ be a blow-up along the curve $G$. Denote by $E_{1}$ the exceptional divisor on $X_{1}$. One may write

$$
\pi^{*}\left(Z_{0}\right)=Z_{1}+m E_{1}
$$

where $Z_{1}$ is the strict transform of $Z_{0}$ and $m \geq 2$, because $G$ belongs to singular locus of $Z_{0}$.

We consider the following commutative diagram:

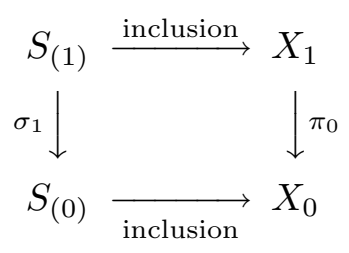


where $S_{(0)}=\pi_{0}^{-1}(F)$ and $S_{(1)}=\pi_{1}^{-1}\left(S_{(0)}\right)$. Denote by $Z_{1} \subset X_{1}$ the strict transform of $Z_{0}$. Then one sees that $Z_{1} \cap S_{(1)}$ is irreducible. Also $\sigma_{1}$ is the blow-up along the center $\left\{G \cap S_{(0)}\right\}$. Now we have

$$
\begin{aligned}
\sigma_{1}^{*}(C) & =\sigma_{1}^{*}\left(Z \cap S_{(0)}\right)=\left.\pi_{0}^{*}(Z)\right|_{S_{(1)}} \\
& =\left.Z_{1}\right|_{S_{(1)}}+\left.m E_{1}\right|_{S_{(1)}} .
\end{aligned}
$$

Because $m \geq 2$, we see that $G$ actually passes through a singular point of $C$. Since $C$ has at most double points, $m \leq 2$. Thus $m=2$ and $\left.E_{1}\right|_{S_{(1)}}$ is either an irreducible $(-1)$-curve or a sum of two distinct $(-1)$-curves. This also means that, on $X_{0}$, one has $1 \leq G \cdot S_{(0)} \leq 2$.

Now it is clear that $Z$ has at most two dictinct horizontal singular curves like $G$ and the multiplicity of each is 2 . The lemma is proved.

Based on the above argument, it is actually clear for us to illustrate all possibilities. Explicitly we have the following possibilities:

I) if $Z_{1}$ is still singular along certain curve over $G$, then $C_{1}$ is still singular and $C$ must have only one cusp (of type $x^{2}=y^{5}$ ). In this case, $G$ is the only singular curve of $Z$ and $Z_{1}$ is singular along only one curve;

II) if $Z_{1}$ is smooth at generic points of $Z_{1} \cap E_{1}$ and the natural map $\left\{\left.E_{1}\right|_{Z_{1}}\right\}_{\text {red }}$ $\mapsto G$ is not birational, then $C$ has a node at each point of $\{G \cap F\}$.

III) if $Z_{1}$ is smooth at generic points of $Z_{1} \cap E_{1}$ and the natural map $\left\{\left.E_{1}\right|_{Z_{1}}\right\}_{\text {red }}$ $\mapsto G$ is birational, then $C$ has a cusp (of type $x^{2}=y^{3}$ ) at each point $\{G \cap F\}$.

Lemma 2.10. For Case II), one has the same inequalities as in 2.7.

Proof. If we are at Case II), then $\pi^{*}\left(K_{X}\right)\left|S=\pi^{*}(Z)\right|_{S}$ always contains an elliptic cycle $C_{0}+C_{1}$ with $g\left(C_{0}\right)=g\left(C_{1}\right)=0$ and $C_{0} \cdot C_{1}=2$ for a general fiber $S$. This means, for any $j$, one has $r_{j} \geq 3$ by Lemma 2.6. We then take $n=0$ and get the same inequalities as in 2.7 .

Combining all arguments above, we are left the following 3 situations derived from possibilities I) and III):

A) $Z$ has only one horizontal singular curve $G, C$ has only one cusp (of type $x^{2}=y^{5}$ ) for a general fiber $F$ and $G$ meets the singular point of $C$;

B) $Z$ has only one horizontal singular curve $G, C$ has exactly 2 cusps (of type $x^{2}=y^{3}$ ) for a general fiber $F$, and $G$ meets the 2 singular points of $C$;

B') $Z$ has two distinct horizontal singular curves $G$ and $H, C$ has exactly 2 cusps (of type $x^{2}=y^{3}$ ) for a general fiber $F$, and both $G$ and $H$ meet one singular point each of $C$.

2.11. Embedded resolution of Type A). We construct an embedded resolution $\pi_{A}$ for the pair $(X, Z)$ of Type A). Take $\pi_{0}$ and $\pi_{1}$ to be as in the proof of Claim 2.9. Because $Z_{1}$ still has a unique singular curve which is over $G$, we denote such a curve by $G_{1}$. Modulo negligible modifications, one may assume $G_{1}$ to be again nonsingular.

Let $\pi_{2}: X_{2} \longrightarrow X_{1}$ be the blow-up along $G_{1}$. Denote by $Z_{2}$ the strict transform of $Z_{1}$ and by $E_{2}$ the exceptional divisor. Set $S_{(2)}=\pi_{2}^{-1}\left(S_{(1)}\right)$. Denote 
by $\sigma_{2}: S_{(2)} \longrightarrow S_{(1)}$ the respective blow-up. Because of the singularity type of $C$, one sees that the strict transform $C_{2}$ of $C$ is already smooth. Since $E_{2}$ only touches $C_{2}$ at one point, we denote by $G_{2}$ the reduced part of $\left.E_{2}\right|_{Z_{2}}$ which is of course irreducible. By considering the multiplicity of $G_{2}$ in $Z_{2}$ (for instance taking blowing-ups and then considering its impacts on $C_{2}$ ), one may see that $Z_{2}$ is smooth at generic points of $G_{2}$. Modulo negligible modifications, $Z_{2}$ is already smooth. But the pull-back of $Z$ is in general not of normal crossing. We need more blow-ups.

Let $\pi_{3}: X_{3} \longrightarrow X_{2}$ be the blow-up along $G_{2}$ which, modulo negligible modifications, is a smooth curve. Let $E_{3}$ be the exceptional divisor and $Z_{3}$ the strict transform of $Z_{2}$. Denote by $S_{(3)}=\pi_{3}^{-1}\left(S_{(2)}\right)$. Then one may see that $E_{2}, E_{3}$ and $Z_{3}$ meet at an irreducible curve $G_{3}$.

Finally blow-up $X_{3}$ along the curve $G_{3}$ (which could be smooth modulo negligible modifications), we get $\pi_{4}: X_{4} \longrightarrow X_{3}$. Denote by $E_{4}$ the exceptional divisor. Take more negligible modifications, we get a resolution $\pi_{A}: X^{\prime} \longrightarrow X$ which is the composition of $\pi_{i}$ and those necessary negligible modifications. We replace our original $\pi$ by $\pi_{A}$, keeping the same notations as above. Pick up a general fiber $S$, then we may see that

$$
\left.\pi^{*}\left(K_{X}\right)\right|_{S}=\left.\pi^{*}(Z)\right|_{S}=10 L_{4}+5 L_{3}+4 L_{2}+2 L_{1}+\tilde{C}
$$

where $L_{i}$ are respective exceptional divisors induced from those blow-ups of $\pi_{i}$ for $i=1,2,3,4$ and $\tilde{C}$ is the strict transform of $C$.

Denote by $\sigma: S \longrightarrow F$ the induced blow-up. Then $\sigma^{*}(C)=\left.\pi^{*}(Z)\right|_{S}$. From the whole process of blow-ups, one sees that $\sigma^{*}(C)$ is a normal crossing divisor on $S$. The intersection graph of $\sigma^{*}(C)$ is as follows:

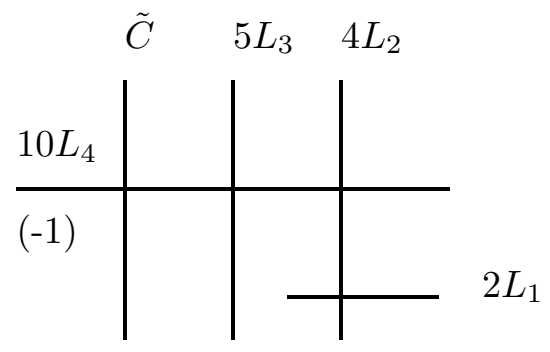

Type A) Slice

Take two joint objects from $\left\{\tilde{Z}, E_{1}, E_{2}, E_{3}, E_{4}\right\}$, they meet a general fiber $S$ at exactly one point. According to the next lemma, the divisor

$$
\tilde{Z}+E_{1}+E_{2}+E_{3}+E_{4}
$$

is normal crossing over a general point of $B$. Taking necessary negligible modifications, $\pi_{A}$ is finally an embedded resolution of $(X, Z)$.

Lemma 2.12. Let $P$ be a point of a smooth variety $V$. Suppose $I$ have $3 \mathrm{ir}$ reducible smooth divisor $H_{i} \subset V$ for $i=1,2,3$ such that $P \in H_{i}$ for all $i$. 
Assume

$$
\left(H_{1} \cdot H_{2} \cdot H_{3}\right)_{P}=1
$$

Then $\mathrm{H}_{1}+\mathrm{H}_{2}+\mathrm{H}_{3}$ is a normal crossing divisor at $P$.

Proof. This is a trivial statement. Denote by $f_{i}$ the local equations of $H_{i}$ for all $i$. Then, by definition,

$$
\operatorname{dim}_{k}\left(\mathcal{O}_{V, P} /\left(f_{1}, f_{2}, f_{3}\right)\right)=\left(H_{1} \cdot H_{2} \cdot H_{3}\right)_{P}=1
$$

This means that $f_{1}, f_{2}$ and $f_{3}$ actually form a local parameters of the point $P$. We are done.

2.13. Embedded resolution of Type $\mathbf{B}^{*}$ ). The construction is somehow similar to 2.11. Both Type B) and Type B') are essentially the same case. We omit those minor differences of the details for Type B) which is simply a copy of the one below. The most important point is that, for Type B) and Type B'), we finally have the same $\left.\pi^{*}(Z)\right|_{S}$ for a general fiber $S$.

Take $\pi_{0}$ to be as in the proof of Claim 2.9. Let $\pi_{1}: X_{1} \longrightarrow X_{0}$ be the blow-up along two smooth curve $G$ and $H$. Denote by $Z_{1}$ the strict transform of $Z$, and by $E_{1}, E_{1}^{\prime}$ the exceptional divisors. Similarly, one may see that $Z_{1}$ is already smooth simply because of the singularity type of $C$. We also see that $E_{1}$ (or $E_{1}^{\prime}$ ) and $Z_{1}$ meet at an irreducible curve $G_{1}$ (or $H_{1}$ ). Modulo negligible modifications, one may assume both $G_{1}$ and $H_{1}$ are smooth curves. We keep parallel notations as in 2.11 .

Going on blow-ups along $G_{1}$ and $H_{1}$, one gets $\pi_{2}: X_{2} \longrightarrow X_{1}$. Denote by $E_{2}, E_{2}^{\prime}$ the exceptional divisors. One sees that $E_{2}$ (or $E_{2}^{\prime}$ ), $E_{1}$ (or $E_{1}^{\prime}$ ) and $Z_{2}$ still meet along an irreducible curve $G_{2}$ (or $H_{2}$ ). One may take negligible modifications such that $G_{2}$ and $H_{2}$ are smooth.

We finally blow-up $X_{2}$ along $G_{2}$ and $H_{2}$ to obtain $\pi_{3}: X_{3} \longrightarrow X_{2}$. Taking further negligible modification, we get a resolution $\pi_{B}: X^{\prime} \longrightarrow X$. We have

$$
\pi_{B}^{*}(Z)=\tilde{Z}+6 E_{3}+6 E_{3}^{\prime}+3 E_{2}+3 E_{2}^{\prime}+2 E_{1}+2 E_{1}^{\prime} .
$$

The slice on a general fiber $S$ is

$$
\left.\pi_{B}^{*}(Z)\right|_{S}=\tilde{C}+6 L_{3}+6 L_{3}^{\prime}+3 L_{2}+3 L_{2}^{\prime}+2 L_{1}+2 L_{1}^{\prime}
$$

where $L_{i}=\left.E_{i}\right|_{S}$ and $L_{i}^{\prime}=\left.E_{i}^{\prime}\right|_{S}$ for all $i$. The intersection graph is as follows. 


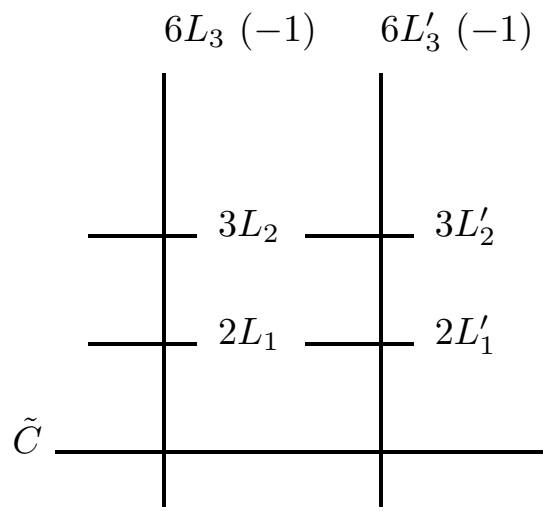

Type B) Slice

Applying Lemma 2.12, one may see that $\pi_{B}$ is an embedded resolution of $(X, Z)$.

2.14. The inequalities for Type A) case. We apply the argument in 2.5. We take two integers $m$ and $n$ such that $1-\frac{n a_{1}}{m a_{1}+s}>\frac{1}{10}$. Write $a:=a_{1} m+s$ as in 2.5. In order to get an effective inequality, we only need to verify the condition $(*)$. For simplicity, we still denote $\pi_{A}$ by $\pi$. Recall that we have

$$
\left.\pi^{*}\left(K_{X}\right)\right|_{S}=\left.\pi^{*}(Z)\right|_{S}=10 L_{4}+5 L_{3}+4 L_{2}+2 L_{1}+\tilde{C}
$$

where $L_{4}^{2}=-1$ and all these curves are smooth rational curves. Set $D_{0}:=$ $\left(\left.\pi^{*}(Z)\right|_{S}\right)_{r e d}$. Then $D_{0}$ is of course a rational tree. From the intersection form of $\left.\pi^{*}(Z)\right|_{S}$, we have

$$
D_{0} \cdot L_{4}=2 \text {. }
$$

Now we verify the condition $(*)$. For a general fiber $S$, we have

$$
\begin{aligned}
& h^{0}\left(S, K_{S}+\left\ulcorner\left.\left(\pi^{*}\left(K_{X}\right)-\frac{n a_{1}}{m a_{1}+s} \pi^{*}(Z)\right)\right|_{S}\right\urcorner\right) \\
\geq & h^{0}\left(S, K_{S}+D_{0}+L_{4}\right)=h^{0}\left(S, K_{S}+\left\ulcorner\left.\frac{1}{5} \pi^{*}(Z)\right|_{S}\right\urcorner\right) \\
= & \frac{1}{2}\left(K_{S}+D_{0}+L_{4}\right)\left(D_{0}+L_{4}\right)+\chi\left(\mathcal{O}_{S}\right)=3 .
\end{aligned}
$$

Thus the inequality (1) gives

$$
K_{X}^{3} \geq 2\left(-2 p_{g}(X)+\delta+\left(2+\frac{n}{m}\right) a+3 h^{2}\left(\mathcal{O}_{X}\right)-3 b-\frac{n(m-1)}{m}+1\right) .
$$

Now we see that, if $p_{g}(X)$ is very big (thus $m$ can be big), then the ratio $K_{X}^{3} / p_{g}(X)$ is close to $\frac{9}{5}$, a very good inequality. The inequality in Theorem 1.1 allows us to assume $p_{g}(X) \geq 5$ and so $a \geq 4$. We may take $m=4$ and $n=3$. Apparently, $1-\frac{3 a_{1}}{4 a_{1}+s} \geq \frac{1}{7}>\frac{1}{10}$ because $s<4$ by definition.

Explicitly, if $b=1$, then we have

$$
K_{X}^{3} \geq \frac{3}{2}\left(p_{g}(X)-3\right) .
$$


This is better than the inequality in Theorem 1.1 only whenever $p_{g}(X) \geq 7$. But the trivial inequality $K_{X}^{3} \geq p_{g}(X)$ amends whenever $p_{g}(X) \leq 6$.

If $b=0$ and $h^{2}\left(\mathcal{O}_{X}\right)=0$, then we have

$$
K_{X}^{3} \geq 2\left(\frac{3}{4} p_{g}(X)-2\right)
$$

This is better than the inequality in Theorem 1.1 .

If $b=0$ and $h^{2}\left(\mathcal{O}_{X}\right)=1$, then we have

$$
K_{X}^{3} \geq \frac{3}{2} p_{g}(X)
$$

This is much better than what we want in Theorem 1.1.

2.15. The inequalities for Type B), Type B') case. We take positive integers $m$ and $n$ such that $1-\frac{n a_{1}}{m a_{1}+s}>\frac{1}{6}$. Write $a:=a_{1} m+s$ as in 2.5. In order to get an effective inequality, we only need to verify the condition $\left(^{*}\right)$. For simplicity, we still denote $\pi_{B}$ by $\pi$. Recall that we have

$$
\left.\pi^{*}\left(K_{X}\right)\right|_{S}=\left.\pi^{*}(Z)\right|_{S}=6\left(L_{3}+L_{3}^{\prime}\right)+3\left(L_{2}+L_{2}^{\prime}\right)+2\left(L_{1}+L_{1}^{\prime}\right)+\tilde{C}
$$

where $L_{3}^{2}=L_{3}^{\prime 2}=-1$ and all these curves are smooth rational curves. Set $D_{0}:=\left(\left.\pi^{*}(Z)\right|_{S}\right)_{\text {red }}$. Then $D_{0}$ is of course a rational tree. From the intersection form of $\left.\pi^{*}(Z)\right|_{S}$, we have

$$
D_{0} \cdot L_{3}=D_{0} \cdot L_{3}^{\prime}=2
$$

Now we verify the condition $(*)$. For a general fiber $S$, we have

$$
\begin{aligned}
& h^{0}\left(S, K_{S}+\left\ulcorner\left.\left(\pi^{*}\left(K_{X}\right)-\frac{n a_{1}}{m a_{1}+s} \pi^{*}(Z)\right)\right|_{S}\right\urcorner\right) \\
\geq & h^{0}\left(S, K_{S}+D_{0}+L_{3}+L_{3}^{\prime}\right)=h^{0}\left(S, K_{S}+\left\ulcorner\left.\frac{1}{3} \pi^{*}(Z)\right|_{S}\right\urcorner\right) \\
= & \frac{1}{2}\left(K_{S}+D_{0}+L_{3}+L_{3}^{\prime}\right)\left(D_{0}+L_{3}+L_{3}^{\prime}\right)+\chi\left(\mathcal{O}_{S}\right)=4 .
\end{aligned}
$$

Thus the inequality (1) gives

$$
K_{X}^{3} \geq 2\left(-2 p_{g}(X)+\delta+\left(2+\frac{2 n}{m}\right) a+3 h^{2}\left(\mathcal{O}_{X}\right)-3 b-\frac{2 n(m-1)}{m}+1\right) .
$$

Still one may see that, if $p_{g}(X)$ is bigger, the ratio $K_{X}^{3} / p_{g}(X)$ is close to $\frac{10}{3}$. Under the assumption of $p_{g}(X) \geq 4$, we may take $m=3$ and $n=2$. So

$$
1-\frac{2 a_{1}}{3 a_{1}+s} \geq \frac{1}{5}>\frac{1}{6}
$$

Explicitly, if $b=1$, then we have

$$
K_{X}^{3} \geq \frac{8}{3}\left(p_{g}(X)-2\right) .
$$

This is better than what we want in Theorem 1.1. 
If $b=0$ and $h^{2}\left(\mathcal{O}_{X}\right)=0$, then we have

$$
K_{X}^{3} \geq 2\left(\frac{4}{3} p_{g}(X)-3\right) .
$$

This is also better than what we want in Theorem 1.1.

If $b=0$ and $h^{2}\left(\mathcal{O}_{X}\right)=1$, then we have

$$
K_{X}^{3} \geq \frac{8}{3} p_{g}(X)-2 .
$$

Already these inequalities are better than the one in Theorem 1.1.

2.16. Summary. Comparing what we have got, we may conclude Theorem 1.1.

\subsection{Proof of Corollary 1.4.}

Proof. If $K_{X}^{3}<\frac{3}{2} p_{g}(X)-\frac{9}{2}$, then we have $p_{g}(X) \geq 5$. This means that we have the canonical map. From the argument above, the only possibility is that $X$ is canonically fibred by curves of genus 2 . This inequality is not empty according to Kobayashi's example.

\subsection{Proof of Theorem 1.2}

Proof. If $K_{X}^{3}<2 p_{g}(X)-6$, then one has $p_{g}(X) \geq 5$. We may study the canonical map. Both 2.2 and Theorem 2.3 tell that either $X$ is canonically fibred by curves of genus 2 or $X$ is canonically fibred by surfaces of general type with $\left(c_{1}^{2}, p_{g}\right)=(1,2)$. We study the later case.

We take the induced fibration $f: X^{\prime} \longrightarrow B$ where a general fiber $S$ is a smooth projective surface with $\left(c_{1}^{2}, p_{g}\right)=(1,2)$. Noting that $f_{*} \omega_{X^{\prime} / B}$ is a vector bundle of rank 2 because $p_{g}(S)=2$, we considering the natural projection

$$
p: \mathbb{P}\left(f_{*} \omega_{X^{\prime} / B}\right) \longrightarrow B .
$$

Because $K_{X^{\prime}}+S_{1}+S_{2} \geq K_{X^{\prime}}$, we see that the fibration $f$ rationally factors through $p$. Taking birational modifications, we may have a morphism from $f$ to $p$. Thus we have the following commutative diagram:

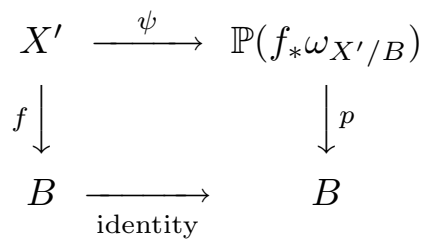

For any fiber $S$ of $f$, we see that $\left.\psi\right|_{S}=\phi_{K_{S}}$ because we have

$$
\left|K_{X^{\prime}}+S_{1}+S_{2} \|_{S}=\right| K_{S} \mid
$$

by Lemma 4.6 of [3], where the $S_{i}$ are general smooth fibers of $f$. Therefore $\psi$ is actually a fibration over a ruled surface with a general fiber a smooth curve of genus 2. We are done. 


\section{Proof of Theorem 1.3}

Though partial effective Noether type of inequalities for a general minimal 3 -fold are given in [3], there remain several hard cases to study. In this section, we are able to develop the technique in [4] to present integral and more precise results which make it possible for us to describe those 3 -folds with small slope $\frac{K_{X}^{3}}{p_{g}(X)}$.

3.1. Notations. We are treating a general object so that most of the divisors we come across are rational divisors. In order to prove Theorem 1.3, we may assume that $X$ is a normal projective minimal 3 -fold with only $\mathbb{Q}$-factorial terminal singularities. We suppose $p_{g}(X) \geq 2$.

We study the canonical map $\varphi_{1}$ which is usually a rational map. Take the birational modification $\pi: X^{\prime} \rightarrow X$, according to Hironaka, such that

(i) $X^{\prime}$ is smooth;

(ii) the movable part of $\left|K_{X^{\prime}}\right|$ is basepoint free. (Sometimes we even call for such a modification that those movable parts of a finite number of linear systems are all basepoint free.)

(iii) $\pi^{*}\left(K_{X}\right)$ is linearly equivalent to a divisor supported by a divisor of normal crossings.

Denote by $g$ the composition $\varphi_{1} \circ \pi$. So $g: X^{\prime} \longrightarrow W^{\prime} \subseteq \mathbb{P}^{p_{g}(X)-1}$ is a morphism. Let $g: X^{\prime} \stackrel{f}{\longrightarrow} B \stackrel{s}{\longrightarrow} W^{\prime}$ be the Stein factorization of $g$. So we have the same commutative diagram as in 2.1. Write

$$
K_{X^{\prime}}={ }_{\mathbb{Q}} \pi^{*}\left(K_{X}\right)+E_{1}={ }_{\mathbb{Q}} M_{1}+Z_{1},
$$

where $M_{1}$ is the movable part of $\left|K_{X^{\prime}}\right|, Z_{1}$ the fixed part and $E_{1}$ an effective $\mathbb{Q}$ divisor which is a $\mathbb{Q}$-sum of distinct exceptional divisors. Throughout we always mean $\pi^{*}\left(K_{X}\right)$ by $K_{X^{\prime}}-E_{1}$. So, whenever we take the round up of $m \pi^{*}\left(K_{X}\right)$, we always have $\left\ulcorner m \pi^{*}\left(K_{X}\right)\right\urcorner \leq m K_{X^{\prime}}$ for all positive number $m$. We may also write

$$
\pi^{*}\left(K_{X}\right)={ }_{\mathbb{Q}} M_{1}+E_{1}^{\prime},
$$

where $E_{1}^{\prime}=Z_{1}-E_{1}$ is actually an effective $\mathbb{Q}$-divisor.

If $\operatorname{dim} \varphi_{1}(X)=2$, we see that a general fiber of $f$ is a smooth projective curve $C$ of genus $g \geq 2$. If $\operatorname{dim} \varphi_{1}(X)=1$, we see that a general fiber $S$ of $f$ is a smooth projective surface $S$ of general type. The invariants of $S$ are $\left(c_{1}^{2}\left(S_{0}\right), p_{g}(S)\right)$ where $S_{0}$ is the minimal model of $S$.

A generic irreducible element $S$ of $\left|M_{1}\right|$ means either a general member of $\left|M_{1}\right|$ whenever $\operatorname{dim} \varphi_{1}(X) \geq 2$ or, otherwise, a general fiber of $f$.

For reader's convenience, we recall known results from [3].

Theorem 3.2. (Theorem 3 and Proposition 3.4 of [3]) Under the above assumptions, one has

1) $K_{X}^{3} \geq 2 p_{g}(X)-4$ whenever $p_{g}(X) \geq 6$, $\operatorname{dim}(B)=2$ and $g(C) \geq 3$;

2) $K_{X}^{3} \geq \frac{3}{2} p_{g}(X)-\frac{5}{2}$ whenever $p_{g}(X) \geq 10,\left(c_{1}^{2}\left(S_{0}\right), p_{g}(S)\right)=(1,1), \operatorname{dim}(B)=$ 1 and $\operatorname{dim} \varphi_{2 K_{X}}(X) \geq 2$. 
Standard surface theory tells us that a surface $S$ of general type with $K_{S_{0}}^{2}=1$ has only 2 possibilities: either $p_{g}(S)=1$ or $p_{g}(S)=2$.

Before proving Theorem 1.3, we must study the other cases. The following proposition presents a general method to estimate certain intersection numbers on $X$.

Proposition 3.3. Let $X$ be a minimal projective 3-fold of general type with only $\mathbb{Q}$-factorial terminal singularities and assume $p_{g}(X) \geq 2$. Keep the same notations as in 3.1. Pick up a generic irreducible element $S$ of $\left|M_{1}\right|$. Suppose, on the smooth surface $S$, there is a movable linear system $|G|$ and denote by $C$ a generic irreducible element of $|G|$. Set $\xi:=\left(\pi^{*}\left(K_{X}\right) \cdot C\right)_{X^{\prime}}$ and

$$
p:= \begin{cases}1 & \text { if } \operatorname{dim} \varphi_{1}(X) \geq 2 \\ a & \text { if } \pi^{*}\left(K_{X}\right) \equiv_{\mathbb{Q}} a S+\text { effective } \mathbb{Q} \text {-divisors }\end{cases}
$$

Assume

(i) there is a rational number $\beta>0$ such that $\left.\pi^{*}\left(K_{X}\right)\right|_{S}-\beta C$ is numerically equivalent to an effective $\mathbb{Q}$-divisor;

(ii) the inequality $\alpha:=\left(m-1-\frac{1}{p}-\frac{1}{\beta}\right) \xi>1$ holds. Set $\alpha_{0}:=\ulcorner\alpha\urcorner$. Then we have the inequality

$$
m \xi \geq 2 g(C)-2+\alpha_{0}
$$

Proof. This is a weak version of Theorem 2.2 in [4]. We do not need the birationality of $\varphi_{m}$. So one may drop additional assumptions there.

3.4. The case $\operatorname{dim}(B)=1$ and $c_{1}^{2}\left(S_{0}\right) \geq 2$. We have

$$
\pi^{*}\left(K_{X}\right)={ }_{\mathbb{Q}} M_{1}+E_{1}^{\prime} \equiv_{\mathbb{Q}} a S+E_{1}^{\prime}
$$

where $a \geq p_{g}(X)-1$. So one has

$$
K_{X}^{3}=\pi^{*}\left(K_{X}\right)^{3} \geq\left(\pi^{*}\left(K_{X}\right)^{2} \cdot S\right)\left(p_{g}(X)-1\right) .
$$

If $b=g(B)>0$, then the movable part of $\left|K_{X}\right|$ is already base point free. Thus one has

$$
\left.\pi^{*}\left(K_{X}\right)\right|_{S}=\sigma^{*}\left(K_{S_{0}}\right) .
$$

Thus $\pi^{*}\left(K_{X}\right)^{2} \cdot S=\left(\sigma^{*}\left(K_{S_{0}}\right)\right)^{2} \geq 2$. So

$$
K_{X}^{3} \geq 2\left(p_{g}(X)-1\right) .
$$

From now on, we assume $b=0$ and $p_{g}(X) \geq 12$. In order to apply Proposition 3.3 , we must find the number $\beta$ and the curve $C$.

Note that $p_{g}(X)>0$ implies $p_{g}(S)>0$. According to [8], we know that $\left|2 K_{S_{0}}\right|$ is base point free. So is $\left|2 \sigma^{*}\left(K_{S_{0}}\right)\right|$. We set $C$ be a general member of $\left|2 \sigma^{*}\left(K_{S_{0}}\right)\right|$. So $C$ is a smooth curve with $\operatorname{deg}\left(K_{C}\right) \geq 12$. According to Step 2 of Proposition 3.3 in [3], we have

$$
\left.\pi^{*}\left(K_{X}\right)\right|_{S} \geq \mathbb{Q} \frac{5}{6} \sigma^{*}\left(K_{S_{0}}\right) .
$$


Thus we may set $\beta=\frac{5}{12}$. Also one may set $p=11$. An initial lower bound for $\xi$ is

$$
\xi \geq \frac{5}{12} C^{2} \geq \frac{10}{3}
$$

Now we may choose $m$ and run Proposition 3.3.

Take $m_{1}=4$. Then $\alpha_{1} \geq\left(3-\frac{1}{11}-\frac{12}{5}\right) \xi \geq \frac{64}{33}$. So $\alpha_{0} \geq 2$. Applying Proposition 3.3, one gets $\xi \geq \frac{7}{2}$.

Take $m_{2}=5$. Then $\alpha_{2}=\left(4-\frac{1}{11}-\frac{12}{5}\right) \xi \geq \frac{581}{110}$. So $\alpha_{0} \geq 6$. Applying Proposition 3.3, one gets $\xi \geq \frac{18}{5}$.

Take $m_{3}=6$. Then $\alpha_{3}=\left(5-\frac{1}{11}-\frac{12}{5}\right) \xi \geq \frac{2484}{275}>9$. So $\alpha_{0} \geq 10$. Applying Proposition 3.3 , one gets $\xi \geq \frac{11}{3}$ which might be the best bound through our method.

So we have $\left(\left.\pi^{*}\left(K_{X}\right)\right|_{S}\right)^{2} \geq \frac{5}{12} \xi \geq \frac{55}{36}>\frac{3}{2}$. Thus we have the inequality

$$
K_{X}^{3} \geq \frac{55}{36}\left(p_{g}(X)-1\right) \text {. }
$$

3.5. The case $\operatorname{dim}(B)=1, K_{S_{0}}^{2}=p_{g}(S)=1$ and $\operatorname{dim} \varphi_{2 k_{X}}(X)=1$. We assume $p_{g}(X) \geq 10$. Considering the induced fibration $f: X^{\prime} \longrightarrow B$, we have $q(X) \leq 1$ and $q(X)-h^{2}\left(\mathcal{O}_{X}\right) \geq 0$ according to [5]. In fact, this case is very simple since $f_{*} \omega_{X^{\prime}}$ is an invertible sheaf while $R^{1} f_{*} \omega_{X^{\prime}}=0$. So we have

$$
\chi\left(\mathcal{O}_{X}\right)=1-q(X)+h^{2}\left(\mathcal{O}_{X}\right)-p_{g}(X) \leq 1-p_{g}(X) .
$$

Applying Reid's plurigenus formula ([20]), one has

$$
\begin{aligned}
P_{2}(X) & \geq \frac{1}{2} K_{X}^{3}-3 \chi\left(\mathcal{O}_{X}\right) \\
& \geq \frac{1}{2} K_{X}^{3}+3 p_{g}(X)-3 .
\end{aligned}
$$

We may remodify our original $\pi$ such that the movable part of $\left|2 K_{X^{\prime}}\right|$ is also base point free. Write

$$
\left|2 K_{X^{\prime}}\right|=\left|M_{2}\right|+Z_{2}
$$

where $M_{2}$ is the movable part. One has

$$
M_{2} \equiv a_{2} S
$$

where $a_{2} \geq P_{2}(X)-1$. Because

$$
2 \pi^{*}\left(K_{X}\right) \geq \mathbb{Q} M_{2},
$$

we have

$$
2 K_{X}^{3} \geq a_{2}\left(\left.\pi^{*}\left(K_{X}\right)\right|_{S}\right)^{2} .
$$

So the key point might be to estimate the number $\left(\left.\pi^{*}\left(K_{X}\right)\right|_{S}\right)^{2}$ which is a rational number.

The base point freeness of $\left|2 \sigma^{*}\left(K_{S_{0}}\right)\right|$ allows us to take $C$ to be a general member of this system. Then $C$ is a smooth curve with $\operatorname{deg}\left(K_{C}\right)=6$. Because

$$
\pi^{*}\left(K_{X}\right) \equiv_{\mathbb{Q}} \frac{a_{2}}{2} S+*,
$$


we may take $p=\left\llcorner\frac{a_{2}}{2}\right\lrcorner \geq 12$. Similarly $\left.5 \pi^{*}\left(K_{X}\right)\right|_{S} \geq_{\mathbb{Q}} 4 \sigma^{*}\left(K_{S_{0}}\right)$ by Step 2 of Proposition 3.3 in [3], we may take $\beta=\frac{2}{5}$. We have $\xi \geq \frac{2}{5} C^{2} \geq \frac{8}{5}$.

Now take $m_{1}=5$. Then

$$
\alpha_{1}=\left(4-\frac{1}{p}-\frac{1}{\beta}\right) \xi>2 .
$$

Proposition 3.3 gives $\xi \geq \frac{9}{5}$.

Take $m_{2}=6$. Then

$$
\alpha_{2}=\left(5-\frac{1}{p}-\frac{1}{\beta}\right) \xi>4 .
$$

Proposition 3.3 gives $\xi \geq \frac{11}{6}$.

Take $m_{3}=7$. Then

$$
\alpha_{3}=\left(6-\frac{1}{p}-\frac{1}{\beta}\right) \xi>6 .
$$

Proposition 3.3 gives $\xi \geq \frac{13}{7}$.

In general, we may get

$$
\xi \geq \frac{2 m_{k}-1}{m_{k}}
$$

for all $m_{k} \geq 8$ by induction. Thus $\xi \geq 2$. This means $\left(\left.\pi^{*}\left(K_{X}\right)\right|_{S}\right)^{2} \geq \frac{2}{5} \xi \geq \frac{4}{5}$. So the inequality (5) becomes

$$
K_{X}^{3} \geq \frac{3}{2} p_{g}(X)-2 .
$$

\subsection{Proof of Theorem 1.3.}

Proof. Assume $K_{X}^{3}<\frac{3}{2} p_{g}(X)-\frac{5}{2}$. Because $K_{X}^{3}>0$, one sees that $p_{g}(X) \geq 2$. So one may always consider the canonical map $\varphi_{1}$. Suppose $p_{g}(X) \geq 12$. According to 2.2 , Theorem 3.2 and the inequalities (4) and (6), $X$ must be either canonically fibred by curves of genus 2 or canonically fibred by surfaces of general type with $\left(c_{1}^{2}, p_{g}\right)=(1,2)$. Then a parallel argument to that in the proof of Theorem 1.2 also works. We are done.

In fact, a combination of [3] and this section may present the following more general result for which we omit the details.

Theorem 3.7. There are two sequences (computable) of positive rational numbers $\left\{a_{k}\right\}$ and $\left\{b_{k}\right\}$ with $k \geq 2$ such that

1) $\frac{4}{3}<a_{k} \leq 2$ for all $k \geq 2$ and $a_{k_{1}} \leq a_{k_{2}}$ whenever $k_{1}<k_{2}$;

2) $\lim _{k \rightarrow+\infty} a_{k}=2$ and $\left\{b_{k}\right\}$ is bounded;

3) for any minimal projective 3-fold $X$ of general type with canonical singularities, set $k:=\left\llcorner\frac{p_{g}(X)-2}{2}\right\lrcorner$. If $K_{X}^{3}<a_{k} p_{g}(X)-b_{k}$ and $p_{g}(X) \notin[2,5]$, then $X$ is fibred by curves of genus 2.

3.8. Examples. The only known examples satisfying the equality in Theorem 1.1 or the assumption of both Theorem 1.2 and Theorem 1.3 were found by M. Kobayashi ([13]). 
3.9. An open problem. If $X$ is a Gorenstein minimal projective threefold of general type with only canonical singularities, then it is well-known that $\chi\left(\mathcal{O}_{X}\right)<0$ according to Miyaoka $([15])$. There should be an analogue of the Noether inequality as in Theorem 1.1 in the form:

$$
K_{X}^{3} \geq-a \chi\left(\mathcal{O}_{X}\right)-b
$$

where $a$ and $b$ are positive rational numbers. One may try to study the bicanonical map of $X$. We have an effective lower bound for $a$. Any bound $a>1$ is nontrivial and interesting. The author's opinion is that to find a Noether inequality in this direction is more difficult simply because the inter relations among $p_{g}, q$ and $h^{2}\left(\mathcal{O}_{X}\right)$ are far from being clear to us, unlike in surface case.

\section{References}

[1] Miguel A. Barja, Lower bounds of the slope of fibred threefolds, International J. Math. 11 (2000), 461-491

[2] F. Catanese, Canonical rings and "special" surfaces of general type. Algebraic geometry, Bowdoin, 1985 (Brunswick, Maine, 1985), 175-194, Proc. Sympos. Pure Math., 46, Part 1, Amer. Math. Soc., Providence, RI, 1987.

[3] Meng Chen, Inequalities of Noether type for 3-folds of general type, J. Math. Soc. Japan 56 (2004), 1131-1155.

[4] Meng Chen, Canonical stability of 3-folds of general type with $p_{g} \geq 3$, Internat. J. Math. 14 (2003), 515-528.

[5] Meng Chen, Complex varieties of general type whose canonical systems are composed with pencils, J. Math. Soc. Japan 51 (1999), 331-335.

[6] Zhijie Chen, On the geography of surfaces. Simply connected minimal surfaces with positive index. Math. Ann. 277 (1987), 141-164.

[7] Zhijie Chen, The existence of algebraic surfaces with preassigned Chern numbers. Math. Z. 206 (1991), 241-254.

[8] C. Ciliberto, The bicanonical map for surfaces of general type, Proc. Symposia in Pure Math. 62 (1997), 57-83.

[9] H. Esnault, E. Viehweg, Lectures on Vanishing Theorems. DMV-Seminar 20 (1992), Birkhäuser, Basel-Boston-Berlin.

[10] B. Hunt, Complex manifold geography in dimension 2 and 3, J. Differential Geom. 30 (1989), 51-153.

[11] Y. Kawamata, A generalization of Kodaira-Ramanujam's vanishing theorem, Math. Ann. 261 (1982), 43-46.

[12] Y. Kawamata, K. Matsuda, K. Matsuki, Introduction to the minimal model problem, Adv. Stud. Pure Math. 10 (1987), 283-360.

[13] M. Kobayashi, On Noether's inequality for threefolds. J. Math. Soc. Japan 44 (1992), 145-156.

[14] J. Kollár, S. Mori, Birational geometry of algebraic varieties, 1998, Cambridge Univ. Press.

[15] Y. Miyaoka, The pseudo-effectivity of $3 c_{2}-c_{1}^{2}$ for varieties with numerically effective canonical classes, Algebraic Geometry, Sendai, 1985. Adv. Stud. Pure Math. 10 (1987), 449-476.

[16] M. Noether, Zur Theorie des eindeutigen Entsprechens algebraischer Ge-bilde, Math. Ann. 2 (1870), 293-316; 8 (1875), 495-533.

[17] K. Ohno, Some inequalities for minimal fibrations of surfaces of general type over curves, J. Math. Soc. Japan 44 (1992), 643-666 
[18] Ulf Persson, An introduction to the geography of surfaces of general type. Algebraic geometry, Bowdoin, 1985 (Brunswick, Maine, 1985), 195-218, Proc. Sympos. Pure Math., 46, Part 1, Amer. Math. Soc., Providence, RI, 1987.

[19] M. Reid, Minimal models of canonical 3-folds, Adv. Stud. Pure Math. 1 (1983), 131-180.

[20] M. Reid, Young person's guide to canonical singularities, Algebraic Geometry, Bowdoin, 1985. Proc. Sympos. Pure Math. 46 Part I, 345-414. Amer. Math. Soc., Providence, RI, 1987.

[21] M. Reid, Quadrics through a canonical surface. Algebraic geometry (L'Aquila, 1988), 191-213, Lecture Notes in Math., 1417, Springer, Berlin, 1990.

[22] E. Viehweg, Vanishing theorems, J. reine angew. Math. 335 (1982), 1-8.

[23] Gang Xiao, An example of hyperelliptic surfaces with positive index. Northeast. Math. J. 2 (1986), 255-257.

[24] Gang Xiao, Surfaces fibres en courbes de genre deux. (French) Lecture Notes in Mathematics, 1137. Springer-Verlag, Berlin, 1985.

[25] S. T. Yau, Calabi's conjecture and some new results in algebraic geometry. Proc. Nat. Acad. Sci. U.S.A. 74 (1977), 1798-1799.

Institute of Mathematics, Fudan University, Shanghai, 200433, PR China

E-mail address: mchen@fudan.edu.cn 